\title{
Liquid-Crystal Phase Transitions Induced by Microtextured Substrates
}

\author{
Tie Zheng Qian* and Ping Sheng \\ Department of Physics, The Hong Kong University of Science and Technology, Clear Water Bay, Kowloon, Hong Kong
}

(Received 11 June 1996)

\begin{abstract}
We show that novel orientational states and temperature-dependent tilt angle of the director may be induced in nematic liquid crystals by microtextured substrates, i.e., spatially mixed alignment potentials. In particular, for an alternating stripe pattern of planar and homeotropic substrate potentials, there is a first-order orientational transition between two states with bulk directors orthogonal to each other. This transition can be observed by varying either the temperature or the texturing periodicity, or through the application of an electric field normal to the substrate. [S0031-9007(96)01750-4]
\end{abstract}

PACS numbers: $64.70 . \mathrm{Md}, 61.30 . \mathrm{Cz}, 68.45 .-\mathrm{v}$

Substrate-induced alignment of liquid crystals is a crucial element in liquid crystal display devices. Up to now, the study of the substrate aligning effect has been focused mostly on uniform substrates. It has been shown that, depending on the aligning potential, a uniform substrate can induce boundary-layer phase transitions, biaxial boundary phases, and other wetting phenomena above the nematic-isotropic phase transition temperature $T_{c}$ [1-5]. Below $T_{c}$, however, the effect of a uniform substrate is mostly to anchor the bulk director, with a slight increase/decrease of the liquid crystalline order close to the substrate.

Some prior studies [6-8] have also examined the effective alignment induced by inhomogeneous substrates or hybrid aligned nematic cell. However, by considering the elastic energy only in the Frank-Oseen form, all these works gave no consideration to elastic relaxational effect that can arise from order parameter variation near the substrate [9]. As a consequence, the elastic correlation length is essentially infinite [10], thus making the effective director alignment the only physical effect that results. In this work, we show that under the more realistic theoretical framework, the existence of a finite elastic correlation length leads to the prediction of novel orientational states and phase transition(s) induced by textured substrates with a spatially mixed pattern of different alignment boundary conditions at the mesoscopic scale i.e., on the order of 0.5 micron. In particular, for a stripe pattern of alternating planar and homeotropic aligning conditions, there can be a first-order phase transition between two bulk orientational states in which one has the director lying in the $y z$ plane, where $z$ is normal to the substrate and $y$ is parallel to the stripes, and the other one aligned along the $x$ direction, shown schematically in Fig. 1. This transition can be observed by varying either the temperature $T$ or the stripe pattern periodicity $P$, or through the application of an electric field $\vec{E}$ in the $z$ direction. In the $y z$ state the tilt angle of the director, $\theta$, as measured from the substrate, can vary between $7^{\circ}$ and $40^{\circ}$ as a function of $T, P$, and $\vec{E}$.
Consider a half space $z \geq 0$ where the substrate, defined to be at $z=0$, is textured into a periodic stripe pattern in the $x$ direction with alternating stripes having either a homeotropic aligning potential, given by $-G_{n} Q_{33} \delta(z)$, or a planar aligning potential, given by $G_{p} Q_{33} \delta(z)$. Here $G_{n}, G_{p}>0$ are the surface potential constants, and $Q_{33}$ is the $z z$ component of the symmetric, traceless tensor order parameter $Q_{i j}$, with the correspondence $1 \leftrightarrow x, 2 \leftrightarrow y$, and $3 \leftrightarrow z$. Here we will consider only the case where the alternating stripes have equal width, with a periodicity $P$. To determine the liquid crystal configuration(s) resulting from the surface alignment potentials, we use the Landau-de Gennes free energy expression [11] as the basis of our calculations. The accuracy of such an approach has recently been verified experimentally [12]. In the

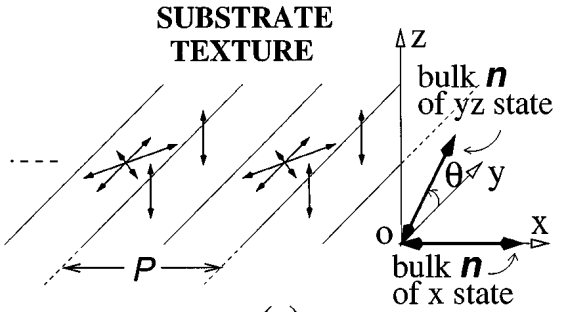

(a)

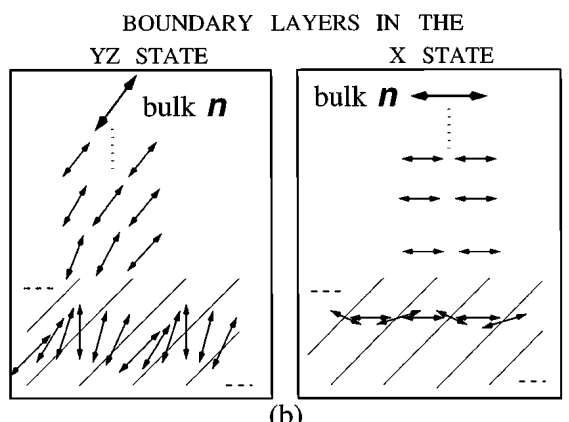

FIG. 1. (a) Schematic illustration of the textured stripe pattern with alternating homeotropic and planar alignment conditions. The directors of the two bulk orientational states are shown at right. (b) The director configurations of the $y z$ state and the $x$ state in the transition boundary layer. 
absence of externally applied field, the total free energy $F$ of the system may be written in the dimensionless form as

$$
\psi=\frac{F}{\Delta^{3} B^{4} / C^{3}}=\psi_{B}+\psi_{S},
$$

where the bulk free energy $\psi_{B}$ and the surface free energy $\psi_{S}$ are given by

$$
\begin{aligned}
\psi_{B}=\int d^{3} \zeta\{ & \left(t+\frac{1}{4}\right)\left(\frac{2}{3} \bar{Q}_{i j}^{2}\right)-\frac{4}{3} \bar{Q}_{i j} \bar{Q}_{j k} \bar{Q}_{k i}+\left(\frac{2}{3} \bar{Q}_{i j}^{2}\right)^{2} \\
& +\frac{2}{3+2 \rho} \bar{Q}_{i j, k}^{2} \\
& \left.+\frac{2 \rho}{3+2 \rho} \bar{Q}_{i j, j} \bar{Q}_{i k, k}\right\} \\
\psi_{S} & =\int d^{3} \zeta\left[g\left(\zeta_{1}\right) \bar{Q}_{33} \delta\left(\zeta_{3}\right)\right] .
\end{aligned}
$$

Here we use the notation where summation over repeated $i$, $j, k$ indices is implied and a comma in the subscript means derivative with respect to the spatial coordinate that follows; $\bar{Q}_{i j}=C Q_{i j} / B$, the length unit $\Delta=\sqrt{L C / B^{2}}$ is the elastic correlation length, which characterizes the distance over which the order parameter can vary significantly, $t=$ $\left(T-T_{C}\right) / 4\left(T_{C}-T^{*}\right)$ with $4\left(T_{C}-T^{*}\right)=B^{2} / a C$ being the temperature unit, $\rho=L_{2} / L_{1}$, and the material constants $a, B, C$, and $L$ are those associated with the usual Landau-de Gennes free energy density $f$ in the uniaxial case, where $S$ is the orientational order parameter,

$$
f=a\left(T-T^{*}\right) S^{2}-B S^{3}+C S^{4}+L(\partial S / \partial z)^{2} .
$$

$T_{C}$ and $T^{*}$ have the usual meanings of the nematicisotropic phase transition temperature and the virtual transition temperature (or the supercooling temperature), respectively, and $L=\left(3 L_{1} / 4\right)+\left(L_{2} / 2\right)$. The dimensionless surface coupling constant $g$ is noted to be a function of $x$. Its unit is given by $G=\Delta B^{3} / C^{2}$. For $5 \mathrm{CB}, \quad a=0.065 \mathrm{~J} /$ $\mathrm{cm}^{3} \mathrm{~K}, B=0.53 \mathrm{~J} / \mathrm{cm}^{3}, C=0.98 \mathrm{~J} / \mathrm{cm}^{3}, L=4.5 \times$ $10^{-14} \mathrm{~J} / \mathrm{cm}$, and $\rho \cong 1[3,4]$. Accordingly, we have $\Delta=40 \AA, G=0.614 \mathrm{erg} / \mathrm{cm}^{2}$, and the temperature unit is $4.4 \mathrm{~K}$. In the following, the values of $g_{n}=0.05$ and $g_{p}=0.08$ will be used [13].

The equilibrium state of the system is given by the configuration $\bar{Q}_{i j}(\vec{\zeta})$ which minimizes the total free energy of the system. Numerical minimization of the free energy has been carried out by spatially discretizing the integral, with five independent components of the tensor $\bar{Q}_{i j}$ being treated as independent variables at each spatial point. Fourth-order finite difference scheme was used to discretize the derivatives, and the conjugate gradient method was employed to seek the optimal configuration. In order to make sure that the state obtained is indeed globally optimal, different random starting configurations were used for each run. Except near the transition point, a unique optimal configuration is invariably obtained, in- dependent of the starting configuration. Close to the transition point, the continuation method is used to obtain the desired state, i.e., the optimal configuration at each step is used as the starting configuration for the next step. The continuation method is also used to identify the metastable state(s) away from the transition point. Accuracy of the calculation has been checked by doubling the number of discretization points. Errors on the order of $0.1 \%$ are the norm. However, for the critical values of the transition temperature and the texture periodicity we find errors on the order of $2 \%$.

Every nematic liquid crystal has an intrinsic elastic correlation length on the order of $\Delta$. If $P \leq \Delta$, then the director would be unable to respond to the spatial texturing, and the substrate aligning potential is essentially homogenized to result in either a uniform planar or a uniform homeotropic alignment, depending on whether $g_{p}+g_{n}$ is positive (planar) or negative (homeotropic). However, when $P \geq 10 \Delta$, the $\bar{Q}_{i j}$ configuration for $z>0$ may be divided into two regions. One region, confined to $z$ less than a few $P$, is characterized by strong lateral $(x)$ variation of the order parameters, reflecting the inhomogeneous substrate aligning effect. This region is denoted the boundary layer. Its thickness is noted to be proportional to $P$. The second region, simply denoted the bulk and extends from $z \approx 2 P$ to $\infty$, is characterized by uniform uniaxial alignment. This is dictated by the fact that otherwise the elastic energy would diverge.

The bulk orientational state is determined by the competition between the elastic energy in the boundary layer and the surface alignment potential. When $P$ is large so that the boundary layer elastic energy is small compared with the surface alignment energy, we obtain the so-called $y z$ state as the optimal bulk configuration, shown in Fig. 1 together with a schematic illustration of its transition boundary layer configuration. The fact that the bulk director lies in the $y z$ plane is understandable physically because the twist elastic constant is almost always smaller than the bend and splay elastic constants, thus favoring the $y z$ orientation over the $x z$ orientation. The magnitude of the tilt angle can also be obtained from numerical solution. In the limit of large $P, \theta$ is predicted to approach $\approx 40^{\circ}$. The value of $\theta$ is found to decrease continuously with decreasing $P$ until it reaches the value of about $7^{\circ}$ at the transition, shown below. The maximum value of $\theta$ is found to be relatively independent of the surface potential(s).

As the texturing periodicity $P$ decreases, elastic energy in the boundary layer becomes comparable to the surface alignment energy, and a transition may occur. This is indeed the case found by our numerical solution. In Fig. 2, we plot the bulk director's tilt angles $\theta$ and azimuthal angle $\phi$, defined relative to the $x$ axis, as a function of $P$. The temperature is fixed at $t=-1.0$. It is seen that as $P$ decreases, the tilt angle decreases continuously to zero at some finite value of $P$. However, 


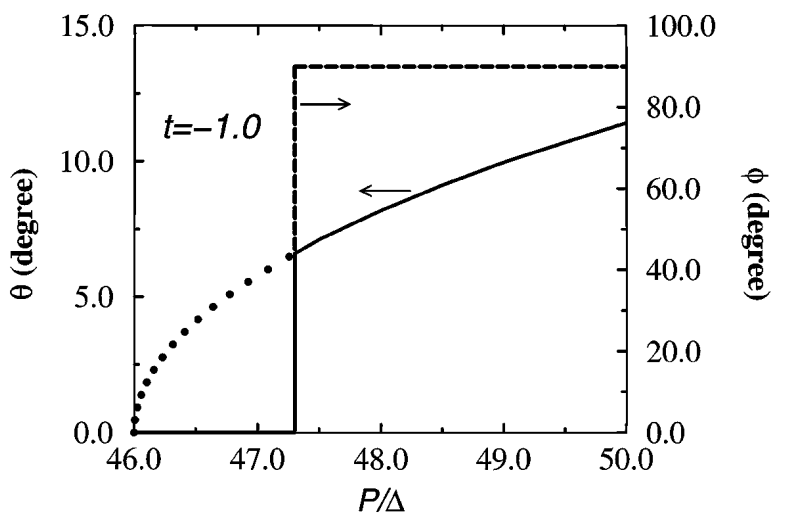

FIG. 2. Variation of the tilt angle $\theta$ and the azimuthal angle $\phi$ plotted as a function of substrate texture periodicity $P$ at fixed $t=-1.0$. Solid line denotes the $\theta$ variation; dashed line denotes the $\phi$ variation. The dotted line denotes the tilt angle variation of the metastable $y z$ state. A first-order phase transition is noted to occur at $P=47.3 \Delta$.

before it can hit zero, a first-order transition occurs at $P_{C}=47.3 \Delta$, whereby the bulk director abruptly jumps to the $x$ axis from the $y z$ plane. The director configuration of the so-denoted $x$ state is illustrated schematically in Fig. 1. If $P$ were fixed at some value not too far from $50 \Delta$, the same transition may be observed as a function of temperature. This is shown in Fig. 3, where the tilt angle is plotted as a function of temperature at fixed $P=47.5 \Delta$. A first-order transition is seen to occur at $t=-1.017$, with an entropy change of $\Delta s=$ $9.17 \times 10^{-5} a \Delta B^{2} / C^{2}$. It should be noted that the tilt angle of the director in the $y z$ state varies strongly only near the transition point. Away from the transition, e.g., when $P \gg P_{C}$, the tilt angle is expected to be nearly constant. However, no calculation has been carried out for temperatures very much below $T_{C}$ (for those cases $P \gg P_{C}$ ) due to the fact that the Landau-de Gennes free energy expression becomes inaccurate far below the nematic-isotropic transition point.

As shown schematically in Fig. 1(b), the $x$ state is characterized by very small elastic distortion in the boundary layer, and a very large surface energy in the homeotropic regions of the substrate. In essence, the transition is from an elastic-energy dominated $y z$ state to a surface-energy dominated $x$ state at the critical values of $P$ and $t$. However, the reason the alignment is along the $x$ axis (denoted the $x$ state) rather than the $y$ axis (denoted the $y$ state) is intriguing at first sight. In order to explain this fact, we note that the last term in the integrand of Eq. (1b) $\left(L_{2} \bar{Q}_{i j, j} \bar{Q}_{i k, k}\right)$ is the one that breaks the rotational symmetry about the $z$ axis in the present problem. It is therefore responsible for distinguishing the $x$ state from the $y$ state. Written out explicitly, this elastic energy consists of two nonzero terms, $L_{2}\left[\left(\bar{Q}_{11,1}+\bar{Q}_{13,3}\right)^{2}+\left(\bar{Q}_{31,1}+\bar{Q}_{33,3}\right)^{2}\right]$. From simple physical arguments, it can be shown that the two terms within the two () brackets are always opposite in sign, in either the $x$ or the $y$ state. A larger $\bar{Q}_{13}$, and hence

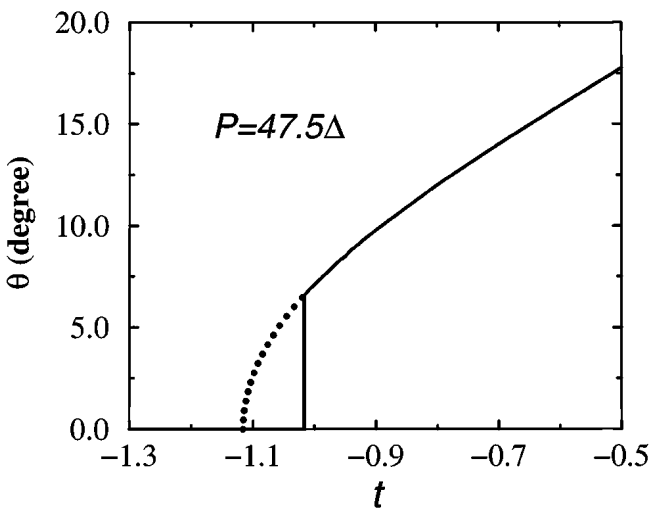

FIG. 3. Variation of the tilt angle $\theta$ and the azimuthal angle $\phi$ plotted as a function of temperature $t$, for fixed $P=47.5 \Delta$. A first-order phase transition is noted to occur at $t=-1.017$. The dotted line denotes the tilt angle variation of the metastable $y z$ state.

an enhanced biaxial character in the boundary layer of the $x$ state, can thus allow not only the "splay-bend" elastic constant to be smaller than the "twist" elastic constant, but also the director to better adjust to the surface potentials in the two adjacent stripes, thereby lowering the surface part of the energy as well. In the limit of $P \rightarrow 0$ the $x$ state is thus the favored configuration.

At above (below) the first-order transition, it is possible to have a field-induced first-order transition provided the dielectric anisotropy is negative (positive). In order to calculate such transitions, it is necessary to add an additional free energy term associated with the external field [11]. In the following we consider only the electric field case; the formalism and results for the magnetic case are expected to be similar. The dimensionless free energy associated with the coupling between the nematic liquid crystal and the electric field is given by

$$
\psi_{E}=\frac{E^{2}}{3 E_{0}^{2}} \int d^{3} \zeta\left[n_{i}^{E} \bar{Q}_{i j}(\vec{\zeta}) n_{j}^{E}\right],
$$

where $n_{i}^{E}$ denotes the $i$ th component of the unit vector along the direction of the applied electric field $\vec{E}$, and

$$
E_{0}=\sqrt{\frac{4 \pi B^{3}}{\left|\delta \varepsilon_{a}\right| C^{2}}}
$$

is the unit of field strength, with $\delta \varepsilon_{a}$ being the maximum dielectric anisotropy. In order to be concrete, we consider the nematic liquid crystal to be in a cell of thickness $D$, sandwiched between two similarly textured substrates. Since the total field energy is on the order of $E^{2} A D=$ $V^{2} A / D$ where $A$ denotes the area of the cell, a fieldinduced transition would occur only when $V^{2} A / D \approx$ const $\times G A$, where $G$ is the surface potential. That means $V_{C} \propto \sqrt{D}$. This should be contrasted with the Freedericksz transition, where the transition voltage $V_{F}$ is independent of the cell thickness. We define $V_{F}=$ 
$\sqrt{4 \pi K_{\text {twist }} /\left|\delta \varepsilon_{a}\right|}$ and $D_{F}$ to be the cell thickness below which $V_{C}<V_{F}$. In terms of the length and electric field units, $D_{F}=2.2 \times 10^{3} \Delta$ and $V_{F}=9 \times 10^{-4} E_{0} D_{F}$. For $5 \mathrm{CB}, D_{F} \approx 9 \mu \mathrm{m}$ and $V_{F} \approx 3 \mathrm{~V}$. In Fig. 4 , we plot the phase diagram in terms of $D_{F} / D$ and $V / V_{F}$ for the case where $\delta \varepsilon_{a} \approx-0.1$ [14], $t=-1.0, P=50 \Delta$, and the field-induced switching is from the $y z$ state to the $x$ state. The upper right region in Fig. 3 is the $x$ state, and the solid line represents the first-order transition which separates the $x$ state from the starting $y z$ state. It should be noted that when the starting state is sufficiently close to the first-order transition point, there can be bistability; i.e., the cell may not switch back to the $y z$ state after the field is removed. This point will be elaborated elsewhere.

The field-induced transition might seem physically impossible if one compares the field coherence length with the boundary layer thickness and realizes the former to be much larger than the latter. This would indeed be the case for strong substrate anchoring. In the present case, however, the elastic energy stored in the boundary layer of the $y z$ state is close to the surface energy of the $x$ state, and it is only necessary for the electric field to supply the energy difference in order for the transition to occur. This is, the effective anchoring is weak. It follows that microtexturing offers a systematic approach to continuously vary the effective anchoring strength from the strong (far away from the transition point) to the weak (close to the transition point). Implications of this approach are presently being pursued.

It should be noted that what we have described in this paper by no means exhausts all possible orientational states and phase transitions that can be induced by different microtexturings. They only serve to illustrate the possibilities associated with a new class of substrates and their induced effects.

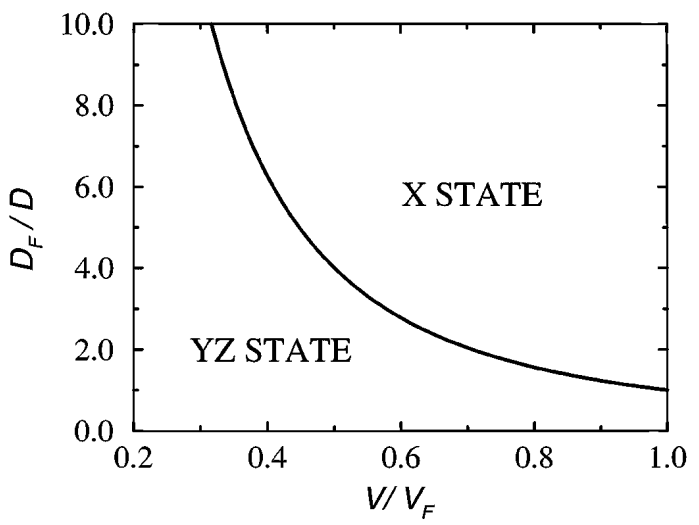

FIG. 4. Phase diagram of the two bulk orientational states plotted as a function of applied voltage $V$ and the inverse cell thickness $D_{F} / D$. $V_{F}$ and $D_{F}$ are defined in the text.
One of us (T.Z.Q.) is supported by the Hong Kong ITDC Grant No. AF/155/94. We wish to thank H. S. Kwok, M. Loy, and Y. Liao for helpful discussions.

*Present address: Institute of Theoretical Physics, The Chinese Academy of Sciences, Beijing, People's Republic of China.

[1] P. Sheng, Phys. Rev. Lett. 37, 1059 (1976).

[2] K. Miyano, Phys. Rev. Lett. 43, 51 (1979).

[3] P. Sheng, Phys. Rev. A 26, 1610 (1982).

[4] T. J. Sluckin and A. Poniewierski, Phys. Rev. Lett. 55, 2907 (1985); P. Sheng, B.Z. Li, M. Y. Zhou, T. Moses, and Y.R. Shen, Phys. Rev. A 46, 946 (1992); Y. L'vov, R. M. Hornreich, and D.W. Allender, Phys. Rev. E 48, 1115 (1993); N. Kothekar, D. W. Allender, and R. M. Hornreich, Phys. Rev. E 49, 2150 (1994).

[5] H. Hsiung, Th. Rasing, and Y.R. Shen, Phys. Rev. Lett. 57, 3065 (1986); W. Chen, L. J. Martinez-Miranda, H. Hsiung, and Y.R. Shen, Phys. Rev. Lett. 62, 1860 (1989).

[6] D. Berreman, Phys. Rev. Lett. 28, 1683 (1972).

[7] S. Faetti, Phys. Rev. A 36, 408 (1987).

[8] G. Barbero et al., J. Phys. II (France) 2, 2011 (1992); G. Barbero and R. Barberi, J. Phys. (Paris) 44, 609 (1983).

[9] A somewhat different case is that of the study by B. Jerome et al., Phys. Rev. Lett. 71, 758 (1993), where they showed that a bulk orientational transition can be induced by a transition in the monolayer of two types of coadsorbed molecules on the substrate.

[10] In a field theory with the free energy density of the form $L(\nabla \phi)^{2}+m \phi^{2}$, one can define a characteristic length $\sqrt{L / m}$. By considering only the gradient term, the Frank-Oseen theory essentially let $m \rightarrow 0$ so that the characteristic length $\rightarrow \infty$.

[11] P. Sheng and E. Priestley, in Introduction of Liquid Crystals, edited by E. B. Priestley, P.J. Wojtowicz, and P. Sheng (Plenum Press, New York, 1975) p. 153.

[12] X. Zhuang, L. Marrucci, and Y. R. Shen, Phys. Rev. Lett. 73, 1513 (1994).

[13] In Ref. [5], the value of $g_{n}$ obtained is $<0.08$ for 5CB. Our choice is in agreement with their experimental results. The value of $g_{p}$ is not known accurately. However, its effect on the induced orientational states can always be maintained by making the width of the planar stripes proportionally larger (smaller) than the homeotropic stripes if the true value of $g_{p}$ is smaller (larger) than the one chosen.

[14] This is a typical value for the maximum dielectric anisotropy. See, for example, P.G. de Gennes and J. Prost, The Physics of Liquid Crystals (Oxford University Press, New York, 1993). 